and effect on resultant film properties. A. W. Mullendore (Sandia National Laboratories) presented an overview of the history, current methodologies, and applications of the CVD process in "Science and Technology of Chemical Vapor Deposition."

Three materials-specific presentations rounded out this session: "Effect of Film Composition on the Corrosion Behavior of lon-Plated $\mathrm{TiC}^{\prime \prime}$ by $\mathrm{H}$. Yoon and W.B. Carter (Georgia Institute of Technology); "Influence of Surface Preparation on Tritium Permeation into $304 \mathrm{~L}$ Stainless Steel" by D. A. Hardwick (Los Alamos National Laboratory); and the use of "Refractory Metal Ions in Arc Vapor Ion Deposition" for formation of refractory nitride coatings, by C. Bergman (MultiArc). Each of these presentations exemplified state-of-the-art surface modification applications while collectively confirming the wide variety of processes, properties and phenomena that fall within the surface modification field.

\section{Researchers Discuss Advances in Hyperfine Interactions}

During the week of September 8, 1986 more than 250 researchers, including over 100 from abroad, gathered in Bangalore, India, for the 7 th International Conference on Hyperfine Interactions. Hyperfine interactions methods have advanced considerably since the last international conference on this topic three years ago, and many presentations were devoted to details of new techniques with greater resolution in the time and energy domain and greater use of advanced apparatus such as new lasers, on-line isotope separators at heavy ion accelerators, and so forth.

Hyperfine interactions as a class of techniques can often be a valuable adjunct to the more conventional approach to materials research. Many contributions to the Bangalore conference dealt with such applications. These techniques comprise experiments such as the Mössbauer effect, perturbed angular correlations and distributions of gamma radiation, nuclear magnetic and/or quadrupole resonance, muon spin rotation (se" " $\mu S R-86$ : Report on the 4 th International Conference at Uppsala" by K. Crowe and A.M. Portis in this issue), and nuclear orientation at cryogenic temperatures. The hyperfine interaction itself is the interaction between the electromagnetic moments of the nucleus and the surrounding atomic and band electrons in the environment of the nucleus. Thus, the technique samples the material environment from the microscopic point of view of the nuclear site.

The Bangalore conference was organized by Drs. S.H. Devare, H.G. Devare, and

\title{
Hyperfine Interactions: Lost in America
}

Hyperfine interactions is an excellent example of a field which suffers a lack of identity by virtue of being interdisciplinary. Most students of science will recognize the phrase "hyperfine interactions" as a phenomenon associated with atomic physics. It probably first came to their attention when they were studying the emission or absorption spectra of atoms where the concept of hyperfine structure in optical spectra caused by the interaction of the nuclear spin with the atomic electrons was introduced.

Hyperfine interactions, of course, derives its name from that phenomenon; but as the accompanying conference report illustrates, it is now a technique applied far more broadly than merely in optical spectroscopy. In addition to the materials-related topics described in the conference report, the technique is invaluable in the study of nuclear moments for nuclear physics purposes and in the fundamental study of the electronic structure and magnetic structure of materials.

Examining the demographics of the attendance from outside India at the Bangalore conference reveals that $\mathrm{Eu}$ rope was by far the best represented. From within Europe, West Germany was most evident. This is no accident. In West Germany a funding category within the ministry governing scientific research is labeled Nuclear Solid State Physics or Nuclear Solid State Chemistry. These terms include explicitly the interdisciplinary area covered by hyperfine interactions. In contrast, U.S. government funding agencies are strictly compartmentalized as atomic, nuclear, condensed matter, or materials areas and have no explicit category to support the border region work represented by hyperfine interactions. The work carried on in the United States is primarily funded, if it is accelerator-related, through nuclear physics funding. Similarly, some fraction of the work is related to chemistry departments on university campuses and could be funded through a chemicai sciences budget, but this catch-as-catch-can way of deriving funds for a truly interdisciplinary field has been inadequate. Over the past ten years the practitioners in this field have slowly diffused to fields where greater support may be garnered.
Two subdisciplines of hyperfine interactions, the Mösbauer effect and nuclear magnetic resonance, have somewhat escaped this fate. They existed before the advent of the catchall terminology, "hyperfine interactions," and have developed their own identities within the condensed matter physics and chemistry communities. The Mössbauer effect has demonstrated applications forsteels, and magnetic resonance has been extended into biological and medical applications with substantial impact. In spite of this, other less visible areas of hyperfine interactions have found it difficult to sustain research programs. The dearth of support in this area has gone on solong that students have not been produced in the field, and it is unlikely that a healthy hyperfine interactions program in the United States can be regained.

Perhaps for the hyperfine interactions field (especially in view of the excellent progress being made in Europe and also in Japan), this is no great loss for the scientific community as a whole. But the hyperfine interactions field in the United States may well represent the fate of any crossdisciplinary thrust which does not benefit from explicit recognition at the potential sources of support. There is clearly a continuing need to advise research supporters about the value of a variety of techniques-as obscure as they may appear-and it is incumbent upon not only the practitioners of the techniques, but also those in allied fields to bring this to the attention of those who determine funding directions.

In materials research and developmentby nature interdisciplinary - the analogous problem does not exist as a global one. Funding categories labeled "materials" are present in most funding agencies now. In addition, categories with applications-oriented titles such as electronic materials can also be found. However, the danger is that at the most fundamental level the less popular research directions will be overlooked because they do not fit neatly into an appropriate funding category. Researchers should be encouraged to remain aware that important areas could be overlooked and work to see that the fate of those areas is not that of hyperfine interactions in the United States. E.N. KAUFMANN 